\title{
The relationship between eating attitudes and distress tolerance in obsessive compulsive disorder
}

\author{
RUKIYE AY1, OZGUR AYTAS ${ }^{1}$ \\ ${ }^{1}$ Malatya Training and Research Hospital, Malatya, Turkey. \\ Received: 12/15/2017 - Accepted: 10/10/2018 \\ DOI: 10.1590/0101-60830000000176
}

\begin{abstract}
Objective: The main purpose of this study is to investigate the eating attitudes of obsessive compulsive disorder (OCD) patients, while the secondary purpose is to examine the relationship between eating attitudes and distress tolerance. Methods: The study included 60 OCD patients and 60 healthy individuals as a control group. The data of the study were collected using the Padua Inventory (PE), Eating Attitudes Test (EAT-26), Distress Tolerance Scale (DTS) and Beck Depression Inventory (BDI). Results: In comparison to the control group, the EAT-26 $(\mathrm{p}=0.001)$ and BDI $(\mathrm{p}=0.001)$ scores of the patient group were significantly higher, while the DTS total score ( $\mathrm{p}=0.001)$ was significantly low. The patients were divided into two groups based on the EAT-26 cutoff score. In the group with EAT-26 $\geq 30$; the total PI score $(p=0.035)$, rumination $(p=0.010)$, impulses $(p=0.001)$ and sub-scale scores and BDI scores $(p=0.038)$ were significantly higher, while the DTS total score $(\mathrm{p}=0.005)$, tolerance $(\mathrm{p}=0.000)$, regulation $(\mathrm{p}=0.013)$ and self-efficacy $(\mathrm{p}=0.009)$ sub-scale scores were significantly lower. Discussion: Our study found that the eating habits of the OCD patients were more irregular than those of the healthy individuals. Further, the distress tolerance of the patients with irregular eating attitudes was significantly lower.
\end{abstract}

Ay R et al. / Arch Clin Psychiatry. 2018;45(6):139-42

Keywords: Obsessive compulsive disorder, eating attitude, distress tolerance.

\section{Introduction}

The relationship between eating disorders and OCD was proposed about 80 years ago ${ }^{1}$. Some researchers claimed that some symptoms seen in ED are obsessional and called eating disorders, or "modern obsessive-compulsive syndromes" 2 . It was suggested that the symptoms seen in ED, such as repeated checking/seeking for reassurance and ritualistic eating behavior, may have obsessivecompulsive characteristics ${ }^{3}$. In ED, noticeable and regular thoughts about food, body image, and the desire to lose weight are likened to obsessions, while avoiding foods, excessive exercising, ritualistic eating attitudes are liked to compulsions 4 .

The life-long OCD prevalence in patients monitored with an ED diagnosis was found vary from $9.5 \%$ to $62 \%{ }^{5}$. Similarly, the life-long ED prevalence in patients monitored with OCD diagnosis was found vary from $11 \%$ to $42 \%{ }^{6}$. It was argued that the biological mechanism of OCD and ED comorbidity may be serotonin dysregulation that is found in both diseases 7 . In a public study with 407 people, researchers found relationships between neuroticism, and checking and binge eating behavior, while neuroticism was also related to perfectionism and checking, washing, and restrictive eating behavior. As a result, it was suggested that similar clinical symptoms arising from similar personality traits may lead to OCD and ED comorbidity ${ }^{8}$. According to a large-sample genetics study, while the risk of anorexia nervosa (AN) increases 16 folds in female patients with OCD, it increases 32 folds in male patients with OCD. In the study, significantly increased AN risk was also found in first-degree and second-degree relatives of OCD patients, and it was argued that shared, common genes play a role in OCD and ED development, rather than disease-specific genes ${ }^{9}$. However, these findings are not sufficient to explain the relationship between ED and OCD.

Distress tolerance is defined as the capacity for tolerating negative situations. Distress tolerance capacity has mostly been accepted as the capacity for coping with emotional situations ${ }^{10}$. Some studies found that the distress tolerance capacity of OCD patients was lower than individuals without OCD ${ }^{11}$. There are also studies that found a relationship between a reduced distress tolerance capacity and $\mathrm{ED}^{12,13}$. This information raises the question of whether reduced distress tolerance is a mediator in the etiology of the eating disorders seen in
OCD patients. The main purpose of this study is to investigate the eating attitudes of OCD patients, while the secondary purpose is to examine the relationship between eating attitudes and distress tolerance.

\section{Methods}

The study included 60 patients visited the Psychiatry Policlinic of Training and Research Hospital between November 2016 and April 2017, underwent monitoring and treatment, diagnosed with OCD based on the DSM-5 criteria, and complied with the criteria of the study, and 60 healthy volunteers who showed similar sociodemographic characteristics. The research project was approved by the Clinical Practices Ethics Board of Malatya, and written consent was taken from the participants.

While literate OCD patients of age 18 to 65 who volunteered were included in the study, the study excluded patients who had an additional psychiatric disease diagnosis other than OCD, or any neurological or systemic disease that might affect cognitive functions.

Healthy volunteers who were 18-65 years old without a psychiatric diagnosis any neurological or systemic disease that might affect cognitive functions were taken as control group.

\section{Instruments}

\section{Sociodemographic Data Form}

This is a questionnaire developed by the researchers to determine the sociodemographic characteristics of the participants. They were about age, sex, marital status, educational status, work status, place of residence, weight, height, BMI, past psychiatric illness, presence of psychiatric illness in the family. The BMI is then calculated by dividing the subject's weight by the square of his/her height.

\section{Padua Inventory (PI)}

The PI is a self-report scale developed by Sanavio ${ }^{14}$ in 1988 with the purpose of measuring the distribution and severity of obsessions and compulsions in OCD patients and healthy individuals. While the 
original scale consisted of 60 items, shortened forms consisting of 39 and 41 items were developed later. Each item of the 5-point Likerttype scale is scored in the range of $0-4$. The reliability and validity study of the 41-item version of the scale in Turkish was carried out by Besiroglu et al. ${ }^{15}$. The scale has five sub-scales as cleaning, impulses, checking, rumination, and precision.

\section{Eating Attitudes Test (EAT-26)}

The EAT-26 is a self-report scale designed to investigate potential disorders in the eating behaviors of individuals who have been diagnosed with an ED and those who have not ${ }^{16}$. An adaptation of the scale into Turkish was achieved by Savasir and Erol17. EAT-26 is a 40-item, 6-point Likert-type scale, and its cutoff score is 30 .

\section{Distress Tolerance Scale (DTS)}

The Distress Tolerance Scale was developed in 2005 by Simons and Gaher ${ }^{10}$. The items are scored in the range of 1-5 with the total score in the range of 15-75. High scores indicate high levels of emotional distress tolerance. Sargin et al. ${ }^{18}$ conducted the Turkish standardization work of the scale. There are three sub-scales (1) tolerance, (2) regulation and (3) self-efficacy in the Turkish form of the scale.

\section{Beck Depression Inventory (BDI)}

The BDI is a 4-point Likert-type self-report scale that consists of 21 statements of self-evaluation, developed in 1961 by Beck ${ }^{19}$. The items are scored in the range of 0-3, and amount to a total score of $0-63$. The validity and reliability study of the scale in Turkish were carried out by $\mathrm{Hisli}^{20}$, and the cutoff point of the scale adapted into Turkish was determined as 17 .

\section{Measurement, analysis, statistical analysis methods}

The analysis of the data collected from the patient and control groups was conducted using the "SPSS for Windows 22" statistical package software. The data collected by counting were indicated as percentages, and the data collected by measurement were indicated as means and standard deviations. Categorical data were compared by a chisquared test, numerical variables of the groups were compared by the parametric test of independent t-test and the non-parametric test of Mann-Whitney $U$ test, and the relationship between non-parametric numerical variables was analyzed by the Spearman correlation test. In all analyses, the level of significance was taken as $0.95(\mathrm{p}<0.05)$.

\section{Results}

The 60 OCD patients and 60 healthy individuals as a control group included in the study were compared. No statistically significant difference was found between the groups in terms of age, sex and BMI. In comparison to the control group, the EAT-26 $(\mathrm{p}=0.000)$ and BDI $(\mathrm{p}=0.000)$ scores of the patient group were significantly higher, while the patients' DTS total score $(\mathrm{p}=0.000)$, tolerance $(\mathrm{p}=$ $0.000)$, regulation $(\mathrm{p}=0.000)$, and self-efficacy $(\mathrm{p}=0.000)$ sub-scale scores were significantly lower (Table 1).

The patients were divided into two groups based on their EAT-26 scores as 47 EAT- $26<30$ patients $(72.4 \%)$, and 13 EAT $\geq 30$ patients (27.6\%), and compared. There was no significant difference between the groups in terms of age, sex, BMI, and the PI sub-scales of washing, checking and precision. In the group with EAT- $26 \geq 30$; the total PI score $(\mathrm{p}=0.035)$, rumination $(\mathrm{p}=0.010)$, impulses $(\mathrm{p}=0.001)$ subscale scores and BDI scores $(\mathrm{p}=0.038)$ were significantly higher, while the DTS total score $(\mathrm{p}=0.005)$, tolerance $(\mathrm{p}=0.000)$, regulation $(\mathrm{p}=$ $0.013)$ and self-efficacy $(\mathrm{p}=0.009)$ sub-scale scores were significantly lower (Table 2). There was weak correlations between the EAT-26 score and the scores of the rumination sub-scales of PI ( $r: 0.277)$ and there were medium-level correlations between the EAT-26 score and the PI total score (r: 0.430), the sub-scales of the impulses ( $\mathrm{r}: 0.370$ ), and BDI scores ( $r: 0.467)$, and the self-efficacy sub-scale of DTS ( $\mathrm{r}:-0.456)$, and there were strong correlations between the EAT-26 score and the DTS total score (r: -0.729), tolerance ( $\mathrm{r}:-0.670)$ and regulation ( $\mathrm{r}$ :-0.555) (Table 3). Partial correlation analysis was carried out to examine the effects of distress tolerance on eating attitude independently of the scores of depression and obsessive-compulsive symptoms. It was seen that, independently of the PI total score, subscale scores and BDI scores, the strong negative correlation between the DTS total scores and EAT-26 scores continued ( $\mathrm{r}$ : -0.580).

Table 1. Sociodemographic and clinical characteristics according to OCD patients and healty controls

\begin{tabular}{|l|c|c|c|}
\hline & OCD (n: 60) & Control (n: 60) & $p$ \\
\hline Age & $31.33 \pm 9.44$ & $31.10 \pm 6.92$ & 0.878 \\
\hline Gender & $31 / 29$ & $31 / 29$ & 1.000 \\
\hline BMI & $25.55 \pm 3.01$ & $24.99 \pm 2.28$ & 0.253 \\
\hline EAT-26 & $21.21 \pm 13.39$ & $11.80 \pm 4.80$ & 0.000 \\
\hline DTS total & $37.25 \pm 9.43$ & $51.36 \pm 9.38$ & 0.000 \\
\hline Tolerance & $19.7 \pm 7.17$ & $31.25 \pm 8.04$ & 0.000 \\
\hline Regulation & $8.81 \pm 3.06$ & $11.38 \pm 2.86$ & 0.000 \\
\hline Self-efficacy & $8.65 \pm 1.58$ & $10.06 \pm 2.15$ & 0.000 \\
\hline BDI & $18.53 \pm 9.39$ & $10.15 \pm 5.80$ & 0.000 \\
\hline
\end{tabular}

OCD: obsessive compulsive disorders; EAT-26: Eating Attitudes Test; DTS: Distress Tolerance Scale; BDI: Beck Depression Inventory.

Table 2. Sociodemographic and clinical characteristics according to the EAT-26 $<30$ and EAT-26 $\geq 30$ groups

\begin{tabular}{|l|c|c|c|c|}
\hline & EAT-26 $<30(n: 47)$ & EAT-26 $\geq 30(n: 13)$ & $u$ & $p$ \\
\hline Age & $30.40 \pm 9.39$ & $34.69 \pm 9.21$ & 222.000 & 0.133 \\
\hline BMI & $25.42 \pm 3.24$ & $26.01 \pm 2.03$ & 274.500 & 0.578 \\
\hline DTS total & $40.02 \pm 8.56$ & $27.23 \pm 4.28$ & 59.000 & 0.000 \\
\hline Tolerance & $21.68 \pm 6.87$ & $12.92 \pm 2.62$ & 61.000 & 0.000 \\
\hline Regulation & $9.38 \pm 2.81$ & $6.76 \pm 3.13$ & 167.500 & 0.013 \\
\hline Self-efficacy & $8.95 \pm 1.38$ & $7.53 \pm 1.80$ & 163.000 & 0.009 \\
\hline PI total & $59.85 \pm 33.07$ & $79.07 \pm 33.53$ & 188.500 & 0.035 \\
\hline Rumination & $18.85 \pm 8.59$ & $29.38 \pm 12.28$ & 162.000 & 0.010 \\
\hline Cleaning & $15.10 \pm 10.86$ & $15.38 \pm 9.03$ & 288.500 & 0.760 \\
\hline Checking & $14.27 \pm 7.84$ & $16.61 \pm 8.36$ & 268.000 & 0.499 \\
\hline Impulses & $3.80 \pm 3.85$ & $9.76 \pm 5.68$ & 119.500 & 0.001 \\
\hline Precision & $6.10 \pm 5.47$ & $7.92 \pm 7.96$ & 285.500 & 0.718 \\
\hline BDI & $17.14 \pm 8.74$ & $23.53 \pm 10.30$ & 190.000 & 0.038 \\
\hline
\end{tabular}

OCD: obsessive compulsive disorders; EAT-26: Eating Attitudes Test; BMI: body mass index; DTS: Distress Tolerance Scale; PI: Padua Inventory; BDI: Beck Depression Inventory.

Table 3. Correlations between Eating Attitudes Test, Padua Inventory, Distress Tolerance Scale

\begin{tabular}{|l|c|c|}
\hline & \multicolumn{2}{|c|}{ EAT-26 } \\
\hline & $r$ & $p$ \\
\hline DTS total & -0.729 & 0.000 \\
\hline Tolerance & -0.670 & 0.000 \\
\hline Self-efficacy & -0.456 & 0.000 \\
\hline Regulation & -0.555 & 0.000 \\
\hline PI total & 0.430 & 0.001 \\
\hline Rumination & 0.277 & 0.032 \\
\hline Cleaning & 0.044 & 0.740 \\
\hline Impulses & 0.370 & 0.004 \\
\hline Precision & 0.209 & 0.109 \\
\hline Checking & 0.303 & 0.018 \\
\hline BDI & 0.467 & 0.000 \\
\hline
\end{tabular}

EAT-26: Eating Attitudes Test; DTS: Distress Tolerance Scale; PI: Padua Inventory; BDI: Beck Depression Inventory. 


\section{Discussion}

The main purpose of this study is to investigate the eating attitudes of OCD patients, while the secondary purpose is to examine the relationship between eating attitudes and distress tolerance. In our study, eating attitude and depression scores were higher and DTS total scores and sub-scale scores were lower in the patient group. According to the EAT-26 cutoff score, the total PI score and the rumination and impulses sub-scale scores were higher, and the DTS total and sub-scale scores were lower in the group with EAT-26 $\geq$ 30. There was a medium-level negative correlation between distress tolerance and eating attitudes even when the effects of depression and obsessive-compulsive symptoms were statistically controlled.

The first hypothesis of our study is that the eating attitudes of OCD patients will be more irregular than healthy controls. In a study with 30 OCD patients, the rate of eating disorder comorbidity was found as $16.7 \%^{21}$. In another study conducted with 100 OCD patients, EAT-26 was used to reach the conclusion that the ratio of patients with disordered eating behaviors without an ED diagnosis complying completely with the DSM-5 criteria was $11 \%{ }^{22}$. Çelikel et al. ${ }^{23}$ studied 55 OCD patient and healthy controls and found that EAT-26 scores were significantly higher in the patient group. In a study by Tyagi et al. ${ }^{3}$ which used the SCOFF questionnaire, it was found that $22.9 \%$ of 135 patients may have an eating disorder. A clinical analysis based on ICD 10, reported that four of the 135 people fulfilled the criteria for an eating disorder diagnosis. It was reported that, while the ritualistic eating behavior and food-related perfectionism in OCD patients might not fit the diagnosis criteria based on ICD 10, they may be the cause of the ED symptoms found in the questionnaire. In contrast to these studies, there are also studies that did not find eating attitude disorders in OCD patients. EAT-26 was applied on to 16 nonmedicated OCD patients, no patient exceeded the cutoff score of 30 , and eating disorders accompanying OCD were discussed in a more easily detectable fashion with standardized diagnostic interviews ${ }^{24}$. In our study, using the Eating Attitudes Test, we found that the eating attitudes of the OCD patients were significantly more disordered than those of the healthy controls. Thought-action fusion (TAF) is a condition in which, individuals overvalue their adverse ideas, and it is a cognitive bias, which is thought to have a role in the misinterpretation of such ideas. TAF consists of two dimensions, which are TAF-Morality and TAF-Probability ${ }^{25}$. A study by Garcia Soriano et al. ${ }^{26}$ identified obsession-like intrusive thoughts in both obsessive compulsive disorder (OCD) and eating disorders. TAF has been identified as a common cognitive bias, causing emotional disturbances created by intrusive thoughts, in both of these diagnostic identities. In the light of this information, comorbid pathological eating attitudes might have been identified in patients who is included in our study and who use TAF as a cognitive bias. Preventive interventions towards comorbid eating disorders can be performed by working through TAF and by utilizing the results of studies with larger sample sizes, involving assessments with scales specific to TAF. These contradictory results may be explained by the relatively smaller sample, different severity of the disease in the participating OCD patients, symptom patterns, medication, and differences in medication.

The relationship between the presence of eating disorder comorbidity and obsessive-compulsive symptomatology is also an interesting issue. In a study of patients with eating disorders, it was found that AN patients gained higher scores from Padua rumination and Padua impulses sub-scales in comparison to the control group ${ }^{27}$. A study with 100 OCD patients, revealed that, among the dimensions of obsessive-compulsive symptoms, impulse obsessions predicted anorexic eating behavior and ruminations predicted bulimic eating behavior. Items containing impulse obsessions are found to be related to the anxiety of losing control over motor behavior. Items containing rumination are seen to be related to thoughts that the person cannot obstruct even if they want to, difficulty in decision making, and excessive mental effort about situations that are unlikely to happen. Fears of losing control resembles avoidance of food in anorexic patients, while rumination indicates not being able to stop something, therefore not being able to stop episodes of binge eating22. In a study with a non-clinical sample of 270 prospective nurses, it was found that eating attitude is related to obsessive-compulsive symptoms (checking, slowness, rumination sub-scales), and as eating behavior became more disordered, the prevalence of obsessive-compulsive symptoms increased 28 . The symptoms that are commonly seen in eating disorders, include intense thoughts about body image, long eating times and ritualistic eating behaviors such as dividing the food into small pieces, show similarities to obsessive compulsive symptoms. In our study, a significant relationship was found between eating attitude disorders and the sub-scales of rumination and impulses. This agrees with most of the literature. Some cognitive bias similar to TAF-probability may be associated with findings questioned by The Eating Attitudes Test, such as "I have gone on eating binges where I feel that I may not be able to stop", and "I feel that food controls my life", which are the subscales questioning the thought shifting and impulsivity. Pathological eating attitudes and progression to comorbidities with eating disorders can be prevented by interventions towards TAF in individuals with higher thought shifting and impulsivity subscale scores. Different results in studies may be explained by differences among scales used to measure obsessivecompulsive symptoms, or differences regarding whether the samples are clinical or not.

The second hypothesis of our study is that OCD patients with a low distress tolerance will have an eating attitude disorder. To the best our knowledge, based on the literature review, there is not a study concerning this issue with an OCD sample. In a study with a nonclinical sample of 200 participants, it was asserted that deficits in distress tolerance play an important role in the etiology and continuation of bulimic symptoms ${ }^{29}$. Lavender et al. ${ }^{30}$ studied on 93 substance-addicted individuals, and reported that distress tolerance and the expectancy that eating relieves negative effects predicted the development of bulimic symptoms. In a recent study monitoring 206 adolescents for six years the distress tolerance capacity was found to be related to eating disorder symptoms in initial stages, while the risk of developing an eating disorder in later stages was found to be related to basal anxiety, depression and distress tolerance levels ${ }^{12}$. In agreement with most of the literature, our study showed that distress tolerance in the group with eating attitude disorder was significantly lower. However, there are also studies that do not support these findings. In a study on 186 young women, as opposed to expectations, distress tolerance was not found to be related to binge eating behavior. It was discussed that this result may have been caused differences in the sample or differences in evaluation of the symptoms of eating disorder ${ }^{31}$. Different results may have been caused by assessment of eating disorders with different characteristics such a AN, bulimia nervosa and binge eating, and conduction of studies with non-clinical samples. In the future, it may be suitable to plan studies with larger samples where all eating disorder symptoms are investigated on this issue.

Some studies found a significant relationship between increased obsession level and low distress tolerance level11,32-34. It is seen that low emotional distress tolerance has a significant role in the process of resorting to compulsions in neutralizing obsessions that occur by misperception and misinterpretation of sensations. Distress tolerance has a critical role in misinterpretation of threat that is seen in obsessive-compulsive disorder. In contamination obsession, the person perceived microbes as a threat and an anxiety against the possibility of contamination starts to appear. There are negative emotions that accompany anxiety, such as disgust and fear. With these emotions, the person may think they will get ill or make someone ill. At this point, individuals with low distress tolerance levels may resort to more compulsion to neutralize these negative emotions, or show more tendency to avoid situations with possibility of contamination ${ }^{32}$. Low tolerance for negative emotions caused by OCD-like intrusions, leads people with lower levels of distress tolerance to use incompatible strategies to cope with the distress related to the intrusion that they think is unbearable. Additionally, all sub-scales of the Distress Tolerance Scale were found related to neutralization and post-neutralization anxiety ${ }^{35}$. A study of 79 OCD 
and $177 \mathrm{ED}$ patients, concluded that similar intrusive thoughts are found in both disorders, but coping mechanisms are different ${ }^{26}$. In our study, the distress tolerance was determined to be lower in OCD patients with pathological eating attitudes. In these patients, low distress tolerance might have increased the extent of neutralization against intrusive thoughts, by the development of pathological eating attitudes as a way of neutralization. Cognitive interventions to increase distress tolerance in patients with OCD can prevent the development of the pathological eating attitudes.

Based on all this information, low distress tolerance may be one of the mutual etiological reasons for OCD and ED. It may be argued that different clinical pictures are seen due to different coping mechanisms for coping with intrusive thoughts in people with low distress tolerance.

Our study has some limitations. Eating attitude was evaluated by a self-report scale only, and eating disorder diagnosis was not made via clinical interview. However, the eating attitude test used here is a test analyzed for validity and reliability by Garner and Garfinkel ${ }^{16}$. Another limitation is that the test used here investigated anorexia symptoms and did not contain questions about other frequently seen eating disorders such as bulimia and binge-eating disorder; however, the EAT-26 was selected as the most frequently seen ED comorbidity in OCD patients is AN. Another limitation of the study is the nonexclusion of the OCD patients with comorbid conditions such as eating disorders and depression, all of which might be associated with eating attitudes. Additionally, our sample was smaller than those of some studies with non-clinical samples, which may be considered a limitation.

Despite its limitations, our study may contribute to the literature as the first study that investigated the relationship between eating disorders seen in OCD and low distress tolerance. However, OCD and ED are complex clinical situations. The reasons for the prevalence of the comorbidity of these two conditions have not yet been made clear. However, cognitive interventions towards increasing the distress tolerance of OCD patients in the light of these findings, will not only improve the clinical outcomes in OCD, but also prevent ED comorbidity development.

\section{References}

1. Palmer HD, Jones MS. Anorexia nervosa as a manifestation of compulsion neurosis: a study of psychogenic factors. Arch Neurol Psychiatry. 1939;41:856-60.

2. Rothenberg A. Eating disorder as a modern obsessive-compulsive syndrome. Psychiatry. 1986;49(1):45-53.

3. Tyagi H, Patel R, Rughooputh F, Abrahams H, Watson AJ, Drummond L. Comparative prevalence of eating disorders in obsessive-compulsive disorder and other anxiety disorders. Psychiatry J. 2015;2015:186927.

4. Demet MM, Taskın EO. Relationship between obsessive-compulsive disorder and eating disorder. Arch Neuropsychiatry. 2002;39(1):9-19.

5. Godart NT, Flament MF, Perdereau F, Jeammet P. Comorbidity between eating disorders and anxiety disorders: a review. Int J Eat Disord. 2002;32(3):253-70.

6. Micali N, Hilton K, Nakatani E, Heyman I, Turner C, Mataix-Cols D. Is childhood OCD a risk factor for eating disorders later in life? A longitudinal study. Psychol Med. 2011;41(12):2507-13.

7. Jarry JL, Vaccarino FJ. Eating disorder and obsessive-compulsive disorder: neurochemical and phenomenological commonalities. J Psychiatry Neurosci. 1996;21(1):36-48.

8. Pollack LO, Forbush KT. Why do eating disorders and obsessive compulsive disorder co-occur? Eat Behav. 2013;14(2):211-5.

9. Cederlöf M, Thornton LM, Baker J, Lichtenstein P, Larsson H, Rück C, et al. Etiological overlap between obsessive-compulsive disorder and anorexia nervosa: a longitudinal cohort, multigenerational family and twin study. World Psychiatry. 2015;14(3):333-8.

10. Simons JS, Gaher RM. The distress tolerance scale: Development and validation of a self-report measure. Motivation and Emotion. 2005;29(2):83-102.

11. Macatee RJ, Capron DW, Schmidt NB, Cougle JR. An examination of low distress tolerance and life stressors as factors underlying obsessions. J Psychiatr Res. 2013;47(10):1462-8.
12. Juarascio AS, Felton JW, Borges AM, Manasse SM, Murray HB, Lejuez $\mathrm{CW}$, et al. An investigation of negative affect, reactivity, and distress tolerance as predictors of disordered eating attitudes across adolescence. J Adolesc. 2016;49:91-8.

13. Emami AS, Woodcock A, Swanson HE, Kapphahn T, Pulvers K. Distress tolerance is linked to unhealthy eating through pain catastrophizing. Appetite. 2016;107:454-9.

14. Sanavio E. Obsessions and compulsions: the Padua Inventory. Behav Res Ther. 1988;26(2):169-77.

15. Beşiroğlu L, Yücel Ağargün M, Boysan M, Eryonucu B, Güleç M, Selvi Y [The assessment of obsessive-compulsive symptoms: the reliability and validity of the Padua inventory in a Turkish population]. Turk Psikiyatri Derg. 2005;16(3):179-89.

16. Garner DM, Garfinkel PE. The Eating Attitudes Test: an index of the symptoms of anorexia nervosa. Psychol Med. 1979;9(2):273-9.

17. Savasır I, Erol N. The Eating Attitudes Test: an index of the symptome of anorexia nervosa. Psychol Med. 1989;7:19-25.

18. Sargin AE, Ozdel K, Utku C, Kuru E, Alkar OY, Turkcapar MH. Distress tolerance scale: a study of reliability and validity. J Cogn Behav Psychother Res. 2012;1:152-61.

19. Beck AT, Ward CH, Mendelson M, Mock J, Erbaugh J. An inventory for measuring depression. Arch Gen Psychiatry. 1961;4:561-71.

20. Hisli N. Validity and reliability of the Beck Depression Scale for university student. J Psychol. 1989;7:3-13.

21. Jiménez-Murcia S, Fernández-Aranda F, Raich RM, Alonso P, Krug I, Jaurrieta N, et al. Obsessive-compulsive and eating disorders: comparison of clinical and personality features. Psychiatry Clin Neurosci. 2007;61(4):385-91.

22. Abanoz Z. Investigation of eating symptoms in patients of obsessivecompulsive disorders and predicting symptoms of obsessive compulsive on the disordered eating symptoms [tese]. Samsun: Ondokuz Mayıs University; 2008.

23. Çelikel FC, Bingol TY, Yıldırım D, Tel H, Erkorkmaz U. Eating attitudes in patients with obsessive compulsive disorder. Arch Neuropsychiatry. 2009;46:86-90.

24. Joffe RT, Swinson RP. Eating Attitudes Test scores of patients with obsessive-compulsive disorder. Am J Psychiatry. 1987;144(11):1510-1.

25. Piri S, Kabakçi E. [An evaluation of some of the relationships between thought-action fusion, attributional styles, and depressive and obsessivecompulsive symptoms]. Turk Psikiyatri Derg. 2007;18(3):197-206.

26. García-Soriano G, Roncero M, Perpiñá $C$, Belloch $A$. Intrusive thoughts in obsessive-compulsive disorder and eating disorder patients: a differential analysis. Eur Eat Disord Rev. 2014;22(3):191-9.

27. Claes L, Vandereycken W, Vertommen H. Impulsive and compulsive traits in eating disordered patients compared with controls. Personality and Individual Differences. 2002;32:707-14.

28. Usta E, Saglam E, Sen S, Aygin D, Sert H. Eating attitudes and obsessivecompulsive symptoms of nursing students. Journal of Health Science and Profession. 2015;2(2):187-19.

29. Anestis MD, Selby EA, Fink EL, Joiner TE. The multifaceted role of distress tolerance in dysregulated eating behaviors. Int J Eat Disord. 2007;40(8):718-26.

30. Lavender JM, Happel K, Anestis MD, Tull MT, Gratz KL. The interactive role of distress tolerance and eating expectancies in bulimic symptoms among substance abusers. Eat Behav. 2015;16:88-91.

31. Kelly NR, Cotter EW, Mazzeo SE. Examining the role of distress tolerance and negative urgency in binge eating behavior among women. Eat Behav. 2014;15(3):483-9.

32. Schmidt NB, Mitchell M, Keough M., Riccardi C. Anxiety and its disorders. In: Zvolensky MJ, Bernstein A, Vujanovic AA (Eds.). Distress tolerance: theory, research, and clinical applications. New York, NY: The Guilford Press; 2011. p. 105-25.

33. Cougle JR, Timpano KR, Sarawgi S, Smith CM, Fitch KE. A multimodal investigation of the roles of distress tolerance and emotional reactivity in obsessive-compulsive symptoms. Anxiety Stress Coping. 2013;26(5):478-92.

34. Blakey SM, Jacoby RJ, Reuman L, Abramowitz JS. The relative contributions of experiential avoidance and distress tolerance to OC symptoms. Behav Cogn Psychother. 2016;44(4):460-71.

35. Cougle JR, Timpano KR, Fitch KE, Hawkins KA. Distress tolerance and obsessions: an integrative analysis. Depress Anxiety. 2011;28(10):906-14. 